\title{
Levantamento florístico das plantas daninhas em um parque público de Campina Grande, Paraíba, Brasil
}

\author{
Ana Maria Alves da Silva ${ }^{1 *}$ \\ Ivan Dantas Coelho ${ }^{1}$ \\ Paulo Roberto de Medeiros ${ }^{2}$ \\ ${ }^{1}$ Departamento de Biologia, Universidade Estadual da Paraíba \\ Av. das Baraúnas 351, CEP 58100-001, Campina Grande - PB, Brasil \\ ${ }^{2}$ Departamento de Sistemática e Ecologia, Centro de Ciências Exatas e da Natureza \\ Universidade Federal da Paraíba, João Pessoa - PB, Brasil \\ * Autor para correspondência \\ ana.maria.bio@gmail.com
}

Submetido em 25/04/2008

Aceito para publicação em 08/08/2008

\section{Resumo}

As plantas daninhas são caracterizadas pelo seu alto poder de disseminação, o que favorece o seu crescimento em lugares indesejáveis. O presente trabalho fornece uma lista das espécies de plantas daninhas encontradas no Parque da Criança na cidade de Campina Grande, Paraíba, Brasil, a partir de dados coletados entre julho e agosto de 2004. Um total de 67 espécies pertencentes a 18 famílias foi encontrado. Dessas, as famílias Poaceae, Asteraceae, Cyperaceae, Fabaceae, Amaranthaceae, Euphorbiaceae e Malvaceae foram as mais representativas, abrangendo $76 \%$ do total de espécies encontradas. Poaceae e Asteraceae, bastante representativas no parque estudado, estão entre as famílias com maior número de espécies de plantas daninhas no Brasil, compartilhando diversas características que favorecem o seu desenvolvimento. A partir dos dados apresentados no presente trabalho, o controle de ervas daninhas, realizado periodicamente na área de estudo, pode ser implementado com maior eficácia, visto que os processos de controle de plantas daninhas são freqüentemente específicos para cada espécie.

Unitermos: Asteraceae, Brasil, parque público, ervas daninhas, Poaceae

\section{Abstract}

Floristic survey of weeds in a public park of Campina Grande, Paraíba, Brazil. Weeds are renowned by their remarkable seed dissemination, which favors their growth in unwanted places. The present study provides a list of the weed species found in Parque da Criança, Campina Grande, Paraíba, Brazil, from data collected between July and August of 2004. A total of 67 species belonging to 18 families were found. Of these, the families Poaceae, Asteraceae, Cyperaceae, Fabaceae, Amaranthaceae, Euphorbiaceae and Malvaceae were the most representative, grouping $76 \%$ of all species encountered. Poaceae and Asteraceae, which are very representative in the studied park, are amongst the families with the highest number of weed species in Brazil, sharing various features, which favor their development. Based on the data provided in this study, weed control (carried out periodically in the studied area) may be implemented more efficiently, since the processes of controlling weeds are frequently species-specific.

Key words: Asteraceae, Brazil, public park, Poaceae, weeds 


\section{Introdução}

As plantas daninhas são consideradas plantas invasoras que crescem em lugares indesejáveis (Lorenzi, 2000). Essas plantas conseguem se desenvolver rapidamente em diversos locais onde causam prejuízos por competirem com as outras plantas que são do interesse humano (Lorenzi, 1990 e 2000; Holm et al., 1991). Segundo Lorenzi (1990) a competição das plantas daninhas com outras plantas ocorre principalmente devido à sua agressividade e grande produção de sementes com altas capacidades de disseminação e longevidade. Outros fatores que também caracterizam algumas espécies de plantas daninhas são as suas exigências fisiológicas relativamente baixas, as altas taxas de crescimento e as elevadas tolerâncias às variações ambientais (Lorenzi, 1990).

Diversos estudos abordaram a relação entre as plantas daninhas e outras plantas economicamente importantes (Paes e Rezende, 2001; Cardina et al., 2002; Jakelaitis et al., 2003; Murphy et al., 2006; Duarte et al., 2007). Esses estudos tiveram como objetivo central avaliar estratégias potenciais para interromper ou amenizar os impactos causados pelas plantas daninhas em ambientes de utilização humana, principalmente em áreas de culturas agrícolas e em áreas onde as plantas são utilizadas com fins ornamentais. No entanto, as espécies de ervas daninhas precisam ser conhecidas para que prováveis procedimentos de erradicação sejam implementados com maior eficácia localmente, visto que, seu controle, seja ele biológico ou químico, depende de características particulares de cada espécie (Duarte et al., 2007). Além disso, técnicas isoladas apresentam um espectro de ação limitado (Brighenti et al., 2003), o que restringe o controle das plantas daninhas para um número limitado de espécies em uma determinada área. Assim, listas florísticas que são freqüentemente elaboradas (Carvalho e Pitelli, 1992; Duarte e Deuber, 1999; Modesto-Júnior e Mascarenhas, 2001; Erasmo et al., 2004) são importantes fontes de informação para que as estratégias de controle sejam implementadas localmente com maior eficácia. Adicionalmente, as listas fornecem uma base de dados que permite a comparação das espécies de áreas distintas.

Esse trabalho tem por objetivo fornecer uma lista das espécies de plantas daninhas ocorrentes em um parque público localizado na cidade de Campina Grande, Paraíba, Brasil, de forma a auxiliar os procedimentos de erradicação e controle dessas plantas, realizados bimensalmente por órgãos responsáveis.

\section{Material e Métodos}

\section{Área de Estudo}

O Parque da Criança está localizado no centro da cidade de Campina Grande, Estado da Paraíba, Brasil, e apresenta uma área de aproximadamente $67.000 \mathrm{~m}^{2}$ (Figura 1). O parque é um centro onde são desenvolvidas

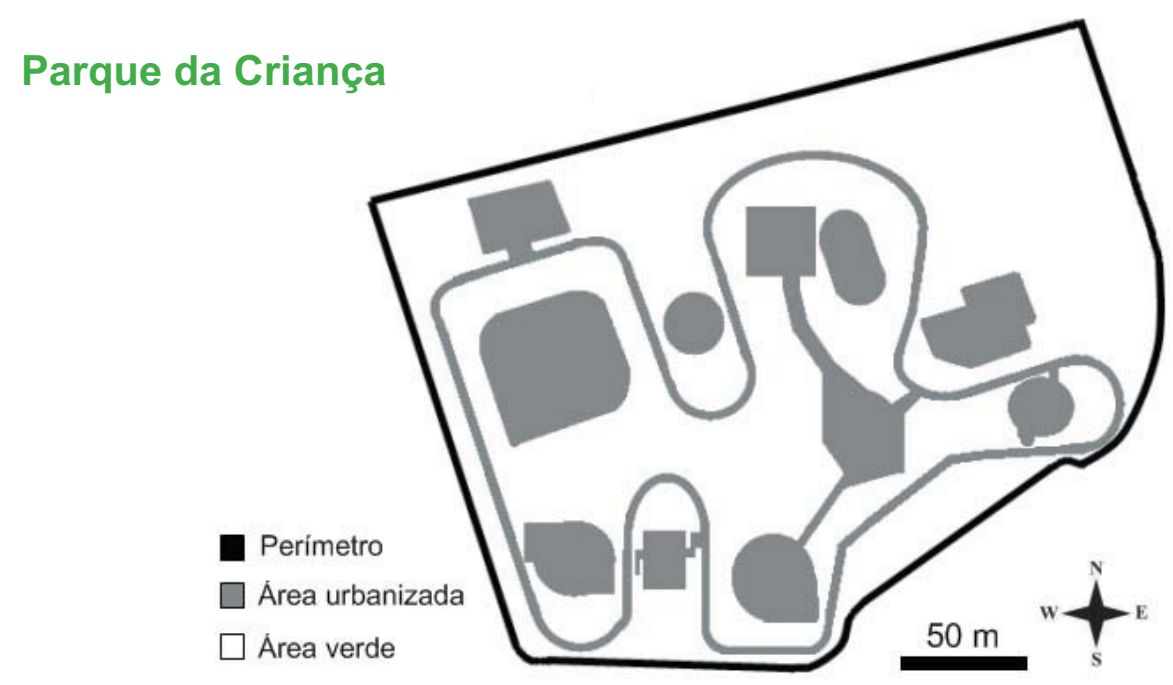

FIGURA 1: Perfil do Parque da Criança localizado no centro da cidade de Campina Grande, Paraíba, Brasil. 
atividades recreativas, sociais e esportivas, apresentando uma grande porção de área verde com 86 espécies de plantas nativas e exóticas distribuídas em 36 famílias que foram plantadas com fins ornamentais. Dentre essas espécies estão entre as mais abundantes: aroeira vermelha (Schinus terebinthifolius Raddi), cássia grande (Cassia grandis L. f.), cássia mimosa (Pithecellobium dulce (Roxb.) Benth.), agave (Agave sisalana Perrine), bromélia (Neoregelia cruenta (Graham) L.B.Sm. e espada-de-SãoJorge (Sansevieria trifasciata Hort. ex Prain.) (ver Lira et al., 2004).

\section{Amostragem}

Entre o período de julho e agosto de 2004 foram realizadas visitas semanais à área de estudo, onde exemplares de plantas daninhas foram coletados em toda extensão de área verde do parque. Grande parte dos exemplares (aproximadamente 60 espécies) foi coletada durante as duas primeiras visitas e, após esse período, as visitas continuaram até que nenhuma nova espécie fosse identificada. Em cada visita, durante um período de aproximadamente três horas, três pesquisadores procuraram exemplares de plantas daninhas que foram coletados e armazenados para posterior identificação. As buscas por plantas daninhas foram planejadas sistematicamente, de forma que toda a abrangência de área verde do parque fosse amostrada igualmente. Com parte do material coletado, exsicatas foram preparadas para registro botânico, sendo que esse material se encontra depositado no Laboratório de Botânica da UEPB.

A identificação das espécies foi realizada no Laboratório de Botânica da Universidade Estadual da Paraíba (UEPB) com base em Lorenzi (1990 e 2000) e Silva e Silva (2007). A nomenclatura usual e a autoria das espécies também se basearam nos referidos autores. Adicionalmente, as espécies que eventualmente não foram identificadas em campo, mas que estavam registradas no laboratório ou em literatura para este parque (e.g. Lira et al., 2004), foram incluídas na lista. A classificação das espécies dentro das famílias foi feita usando como base o sistema APG II, de acordo com Souza e Lorenzi (2005).

\section{Resultados}

Foram encontrados 67 táxons específicos de plantas daninhas distribuídos em 18 famílias (Tabela 1 e Figura 2), das quais 63 foram identificadas até o nível de espécie e quatro foram identificadas até o nível de gênero. As famílias Poaceae, Asteraceae, Cyperaceae, Fabaceae, Amaranthaceae, Euphorbiaceae e Malvaceae foram responsáveis por $76 \%$ da riqueza total, com 51 espécies, enquanto as outras 16 espécies, que representaram os $24 \%$ restantes da riqueza, foram distribuídas dentro de 11 famílias (Figura 3).

TABELA 1: Plantas daninhas registradas no Parque da Criança, Campina Grande, Paraíba.

\begin{tabular}{|l|l|}
\hline \multicolumn{1}{|c|}{ FAMÍLIA/ESPÉCIE } & \multicolumn{1}{c|}{ NOME COMUM } \\
\hline Amaranthaceae & \\
Alternanthera pungens Kunth & Perpétua-do-mato \\
Alternanthera sp. & Budo-diaque \\
Amaranthus spinosus L. & Bredo-de-espinho \\
Amaranthus viridis L. & Alecrim \\
Alternanthera tenella Colla & Caruru-verde \\
\hline Asteraceae & \\
Bidens pilosa L. & Capim-duro \\
Blainvillea biaristata DC. & Picão \\
Centratherum punctatum Cass. & Perpétuo-roxa-do-mato \\
Conyza bonariensis (L.) Cronquist & Rabo-de-raposa \\
Eclipta alba (L.) Hassk. & Erva-botão \\
Emilia sonchifolia (L.) DC. & Serralha-vermelha \\
Emilia coccinea (Sims) G.Don & Pincel-de-estudante \\
Gnaphalium spicatum Lam. & Macela \\
Sonchus oleraceus L. & Serralha \\
Synedrellopsis grisebachii Hieron. \& Kuntze & Agriãozinho \\
Tridax procumbens L. & Erva-de-touro \\
\hline
\end{tabular}




\begin{tabular}{|c|c|}
\hline $\begin{array}{l}\text { Boraginaceae } \\
\text { Heliotropium indicum L. } \\
\text { Heliotropium lanceolatum Ruiz \& Pav. }\end{array}$ & $\begin{array}{l}\text { Fedegoso } \\
\text { Sete-sangrias }\end{array}$ \\
\hline $\begin{array}{l}\text { Brassicaceae } \\
\text { Lepidium virginicum } \mathrm{L} .\end{array}$ & Mastruz \\
\hline $\begin{array}{l}\text { Commelinaceae } \\
\text { Commelina benghalensis L. } \\
\text { Commelina diffusa Burm. f. } \\
\text { Commelina erecta } \mathrm{L} \text {. }\end{array}$ & $\begin{array}{l}\text { Mané-mole } \\
\text { Trapoeraba } \\
\text { Trapoeraba }\end{array}$ \\
\hline $\begin{array}{l}\text { Convolvulaceae } \\
\text { Ipomoea asarifolia (Desr.) Roem. Schult. }\end{array}$ & Salsa \\
\hline $\begin{array}{l}\text { Cyperaceae } \\
\text { Cyperus distans } \mathrm{L} . \mathrm{f} . \\
\text { Cyperus esculentus L. } \\
\text { Cyperus iria } \mathrm{L} . \\
\text { Cyperus meyenianus Kunth } \\
\text { Cyperus rotundus } \mathrm{L} . \\
\text { Cyperus sesquiflorus (Torr.) Mattf. \& Kük. }\end{array}$ & $\begin{array}{l}\text { Tiririca } \\
\text { Tiririca } \\
\text { Tiririca-do-brejo } \\
\text { Tiririca } \\
\text { Tiririca } \\
\text { Tiririca }\end{array}$ \\
\hline $\begin{array}{l}\text { Euphorbiaceae } \\
\text { Chamaesyce hirta (L.) Millsp. } \\
\text { Chamaesyce hyssopifolia (L.) Small } \\
\text { Chamaesyce prostrata (Aiton) Small } \\
\text { Croton glandulosus L. } \\
\text { Phyllanthus niruri L. }\end{array}$ & $\begin{array}{l}\text { Erva-de-santa-luzia } \\
\text { Burra-leiteira } \\
\text { Quebra-pedra-rasteira } \\
\text { Gervão-branco } \\
\text { Quebra-pedra }\end{array}$ \\
\hline $\begin{array}{l}\text { Fabaceae } \\
\text { Desmodium adscendens (Sw.) DC. } \\
\text { Indigofera truxillensis Kunth } \\
\text { Mimosa pudica } \text { L. } \\
\text { Mimosa } \text { sp. } \\
\text { Senna obtusifolia }(\mathrm{L} .) \text { H. S. Irwin \& Barneby } \\
\text { Zornia latifolia } \text { Sm. }\end{array}$ & $\begin{array}{l}\text { Amor-agarrado (feijãozinho) } \\
\text { Anil } \\
\text { Malícia-de-mulher } \\
\text { Maricá } \\
\text { Fedegoso } \\
\text { Urinana }\end{array}$ \\
\hline $\begin{array}{l}\text { Lythraceae } \\
\text { Cuphea sp. }\end{array}$ & Sete-sangrias \\
\hline $\begin{array}{l}\text { Malvaceae } \\
\text { Malvastrum coromandelianum (L.) Garcke } \\
\text { Sida carpinifolia L. f. } \\
\text { Sida linifolia Juss. ex Cav. } \\
\text { Sidastrum micranthum (A. St.-Hil.) Fryxell } \\
\text { Waltheria douradinha A.St.-Hil. }\end{array}$ & $\begin{array}{l}\text { Guanxuma } \\
\text { Malva-baixa } \\
\text { Malva-língua-de-tucano } \\
\text { Malva-preta } \\
\text { Doradinha }\end{array}$ \\
\hline $\begin{array}{l}\text { Nyctaginaceae } \\
\text { Boerhavia diffusa } \mathrm{L} .\end{array}$ & Agarra-pinto \\
\hline $\begin{array}{l}\text { Onagraceae } \\
\text { Ludwigia leptocarpa (Nutt.) H. Hara }\end{array}$ & Cruz de malta \\
\hline
\end{tabular}




\begin{tabular}{|c|c|}
\hline $\begin{array}{l}\text { Poaceae } \\
\text { Brachiaria decumbens Stapf } \\
\text { Cenchrus echinatus L. } \\
\text { Chloris barbata (L.) Sw. } \\
\text { Chloris gayana Kunth } \\
\text { Cynodon dactylon (L.) Pers. } \\
\text { Dactyloctenium aegyptium (L.) Willd. } \\
\text { Digitaria bicornis (Lam.) Roem. \& Schult. } \\
\text { Eleusine indica (L.) Gaertn. } \\
\text { Eragrostis pilosa (L.) P.Beauv. } \\
\text { Paspalum notatum Flüegge } \\
\text { Pennisetum clandestinum Hochst. ex Chiov. } \\
\text { Setaria geniculata (Lam.) P. Beauv. } \\
\text { Sporobolus indicus (L.) R. Br. }\end{array}$ & $\begin{array}{l}\text { Capim-braquiária } \\
\text { Carrapicho-de-agulha } \\
\text { Capim-carrapicho } \\
\text { Capim-pé-de-galinha } \\
\text { Capim-pé-de-galinha } \\
\text { Capim-de-burro } \\
\text { Capim-mão-de-sapo } \\
\text { Capim-pé-de-galinha } \\
\text { Capim-pé-de-galinha } \\
\text { Capim-panasco } \\
\text { Grama-batatais } \\
\text { Capim-quicúo } \\
\text { Capim-rabo-de-raposa }\end{array}$ \\
\hline $\begin{array}{l}\text { Polygalaceae } \\
\qquad \text { Polygala paniculata L. }\end{array}$ & Barba-de-São-Pedro \\
\hline $\begin{array}{l}\text { Portulacaceae } \\
\text { Portulaca oleracea L. } \\
\text { Portulaca } \mathrm{sp} .\end{array}$ & $\begin{array}{l}\text { Onze horas } \\
\text { Beldroega-pequena }\end{array}$ \\
\hline $\begin{array}{l}\text { Rubiaceae } \\
\text { Richardia grandiflora (Cham. \& Schltdl.) Steud. } \\
\text { Spermacoce verticillata L. }\end{array}$ & $\begin{array}{l}\text { Poaia-rasteira } \\
\text { Vassourinha-de-botão }\end{array}$ \\
\hline $\begin{array}{l}\text { Turneraceae } \\
\quad \text { Turnera ulmifolia } \mathrm{L} .\end{array}$ & Chanana \\
\hline
\end{tabular}

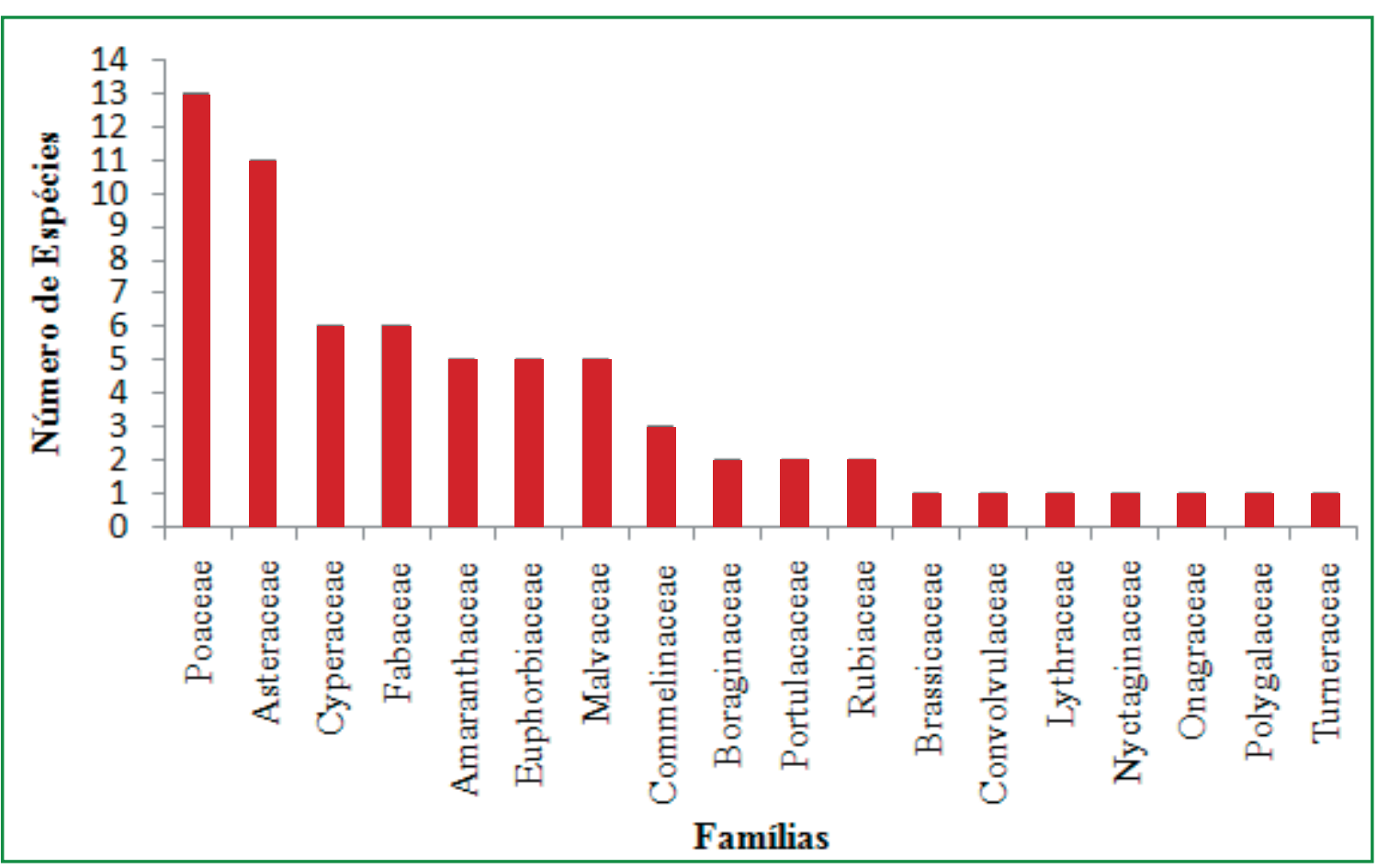

FIGURA 2: Número de espécies de plantas daninhas por família registradas no Parque da Criança, Campina Grande, Paraíba. 


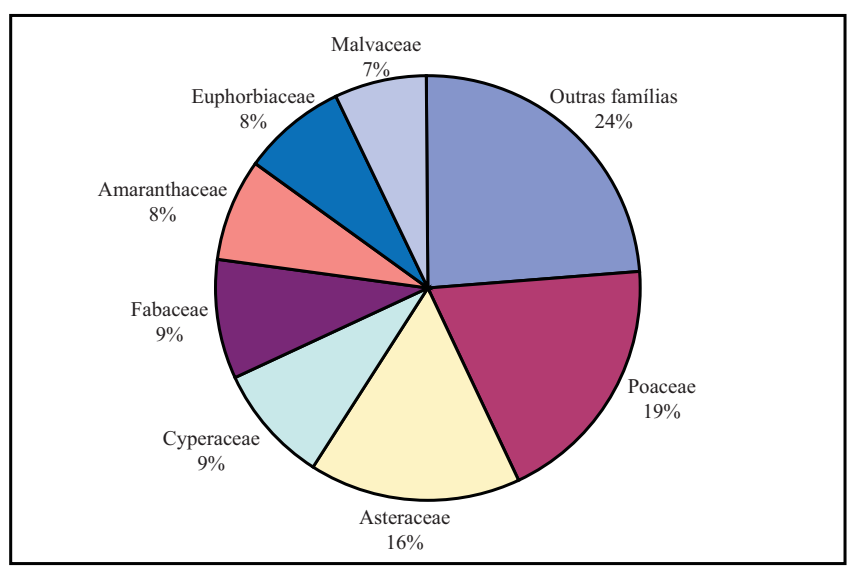

FIGURA 3: Porcentagem das principais famílias de plantas daninhas registradas no Parque da Criança, Campina Grande, Paraíba.

\section{Discussão}

As famílias Poaceae e Asteraceae foram as mais representativas em número de espécies, seguidas por Cyperaceae, Fabaceae, Amaranthaceae, Euphorbiaceae e Malvaceae que também mostraram resultados expressivos. Asteraceae e Poaceae são típicas em estudos com plantas daninhas (e.g. Carvalho e Pitelli, 1992; Duarte e Deuber, 1999; Modesto-Júnior e Mascarenhas, 2001; Paes e Rezende, 2001; Cardina et al., 2002; Jakelaitis et al., 2003; Erasmo et al., 2004; Murphy et al., 2006; Duarte et al., 2007), provavelmente por apresentarem alta disseminação e elevada ocorrência em ambientes antropizados (Pedrotti e Guarim-Neto, 1998).

Poaceae está entre as famílias de plantas mais importantes economicamente no mundo e é, freqüentemente, bastante representativa em termos de espécies de plantas daninhas em vários ambientes (Holm et al., 1991; Lorenzi, 2000; Erasmo et al., 2004). Um estudo desenvolvido por Souza et al. (2003) identificou uma alta interferência de plantas daninhas em agrossistemas de cupuaçuzeiro e pupunheira, sendo que as espécies da família Poaceae foram as mais representativas, assim como o que foi observado no presente estudo. Apesar das áreas amostradas por esses autores possuírem utilidades diferentes das do presente estudo (agricultura versus recreação), a dominância dessa família em áreas tão distintas enfatiza a plasticidade das espécies e, conseqüentemente, sua elevada importância econômica. Segundo Holm et al. (1991), grande parte das espécies da família Poaceae é perene e produz grande quantidade de sementes, o que aumenta consideravelmente o seu poder de disseminação e a colonização de diversos tipos de ambientes, mesmo que suas condições sejam inóspitas.

As espécies da família Asteraceae, quando se tratando de plantas daninhas, estão entre as mais importantes, sendo que algumas dessas espécies são as mais comuns em diversos ambientes do Brasil. Por exemplo, Bidens pilosa, espécie bastante abundante na área estudada (observação pessoal dos autores), é uma planta muito comum, não só no Brasil como em várias outras partes do mundo (Adegas et al., 2003), sendo considerada uma das plantas mais infestantes de culturas anuais e perenes (Kissmann e Groth, 1992). As espécies Asteraceae identificadas no presente estudo apresentam diversas particularidades, como uma biologia generalista e a capacidade de disseminação por várias espécies de insetos (Grombone-Guaratini et al., 2004), o que favorecem o seu crescimento em lugares indesejáveis, principalmente pela sua alta agressividade, o que tem o potencial de inibir o desenvolvimento de outras plantas (Kissmann e Groth, 1992). Assim, as espécies dessa família costumam ser comumente encontradas em vários tipos de ambientes, sejam eles de interesse humano ou não. Segundo Lorenzi (2000), as espécies de Asteraceae estão entre as primeiras plantas daninhas que surgem no campo após o preparo do solo para plantio, o que confirma o seu potencial de desenvolvimento. Além da importância das famílias mencionadas, as seis espécies do gênero Cyperus (Cyperaceae) identificadas no presente estudo também estão entre as plantas daninhas mais importantes do mundo (Holm et al., 1991; Lorenzi, 2000).

As características referentes ao desenvolvimento das plantas daninhas, citadas anteriormente, fazem com que os procedimentos de erradicação adotados para controlar as populações dessas espécies sejam altamente específicos, dependendo de diversos conhecimentos da biologia da cada uma. De fato, para algumas plantas daninhas até o presente momento não existem herbicidas com poder eficaz de erradicação, devido ao fato de suas propriedades biológicas ainda serem desconhecidas (Rodrigues e Almeida, 2005). Dessa forma, como 
evidenciado por Erasmo et al. (2004), uma vez que a composição de plantas daninhas pode variar conforme o ambiente, o conhecimento das espécies existentes nos locais está entre os primeiros passos para realizar o controle populacional dessas plantas.

Os procedimentos de controle, realizados bimensalmente no parque estudado, são feitos deliberadamente. A partir da lista de espécies existentes na área fornecida é possível direcionar os métodos, de forma a maximizar o controle dessas plantas. Apesar da escassez de trabalhos realizados em parques e em locais que possuem fins recreativos ou sociais, sabe-se que a presença de espécies daninhas nesses locais tem o potencial de reduzir a diversidade local, chegando, em casos extremos, a extinguir localmente algumas espécies. Um exemplo do potencial das ervas daninhas está exemplificado no Parque Nacional São Joaquim, em Santa Catarina, Brasil, onde o cardo negro Cirsium vulgare (Savi) Ten. da família Asteraceae ameaça várias espécies nativas pelo seu grande potencial de disseminação (Lima, 2008).

A análise dos resultados permitiu concluir que o número de espécies de plantas daninhas se mostrou bastante expressivo, com espécies comuns em outras localidades do Brasil e do mundo. Assim como o observado em outros estudos, as famílias Poaceae e Asteraceae foram as mais representativas, apesar de que outras famílias também demonstraram números bastante significativos. Essa lista fornece parâmetros comparativos com trabalhos similares e subsídios para a implementação mais eficaz dos processos de controle das plantas daninhas na área.

\section{Agradecimentos}

Os autores são gratos ao Conselho Nacional de Desenvolvimento Científico e Tecnológico (CNPq) e à coordenação do Departamento de Biologia da UEPB pelo auxílio necessário para o desenvolvimento do presente estudo. Agradecemos também a dois consultores anônimos, cujos comentários foram essenciais para o aperfeiçoamento do manuscrito.

\section{Referências}

Adegas, F. S.; Voll. E.; Prete, C. E. C. 2003. Embebição e Germinação de Sementes de Picão-Preto. (Bidens pilosa). Planta Daninha, 21 (1): 21-25.

Brighenti, A. M.; Castro, C.; Gazziero, D. L. P.; Adegas, F. S.; Voll, E. 2003. Cadastramento fitossociológico de plantas daninhas na cultura de girassol. Pesquisa Agropecuária Brasileira, 38 (5): 651-657.

Cardina, J.; Herms, C. P.; Doohan, D. J. 2002. Crop rotation and tillage system effects on weed seedbanks. Weed Science, 50: 448460.

Carvalho, S. L.; Pitelli, R. A. 1992. Levantamento e análise fitossociológica das principais espécies de plantas daninhas de pastagens da região de Selvíria (MS). Planta Daninha, 10 (1): 25-32.

Duarte, A. P.; Deuber, R. 1999. Levantamento de plantas infestantes em lavouras de milho "safrinha" no Estado de São Paulo. Planta Daninha, 17 (2): 297-307.

Duarte, A. P.; Silva, A. C.; Deuber, R. 2007. Plantas infestantes em lavouras de milho safrinha, sob diferentes manejos, no médio Paranapanema. Planta Daninha, 25 (2): 285-297

Erasmo, E. A. L.; Pinheiro, L. L. A.; Costa, N. V. 2004. Levantamento fitossociológico das comunidades de plantas infestantes em áreas de produção de arroz irrigado cultivado sob diferentes sistemas de manejo. Planta daninha, 22 (2): 195-201.

Holm, L. G.; Pancho, J. V.; Herberger, J. P.; Plucknett, D. L. 1991. The world's worst weeds - distribution and biology. $2^{\text {nd }}$ ed. Krieger Publishing Company, Malabar, USA, 609pp.

Jakelaitis, A.; Ferreira, L. R.; Silva, A. A.; Agnes, E. L.; Miranda, G. V.; Machado, A. F. L. 2003. Dinâmica populacional de plantas daninhas sob diferentes sistemas de manejo nas culturas de milho e feijão. Planta Daninha, 21 (1): 71-79.

Grombone-Guaratini, M. T.; Solferini, V. N.; Semir, J. 2004. Reproductive biology of Bidens L. (Asteraceae). Scientia Agricola, 61 (2): 185-189.

Kissmann, K. G.; Groth, D. 1992. Plantas infestantes e nocivas. $2^{\mathrm{a}}$ ed. Basf Brasileira, São Paulo, Brasil, 798pp.

Lima, J. 2008. Cardo-negro ameaça espécies do Parque Nacional de São Joaquim. Disponível em $<$ http://webventureuol.uol. com.br/>. Acesso em 22 de abril de 2008.

Lira, R. S.; Dantas, C. I.; Cavalcanti, F. L. M.; Barros, B. J. M.; Lira, M. V.; Carneiro, T. P. 2004. Diagnóstico paisagístico do Parque da Criança em Campina Grande, PB. Revista de Biologia e Ciências da Terra, 4 (1): 1-23.

Lorenzi, H. 1990. Manual de identificação e controle de plantas daninhas: Plantio direto e convencional. $3^{\mathrm{a}}$ ed. Plantarum, Nova Odessa, Brasil, 269pp.

Lorenzi, H. 2000. Plantas daninhas do Brasil: Terrestres, aquáticas, parasitas e tóxicas. $3^{\mathrm{a}}$ ed. Plantarum, Nova Odessa, Brasil, $620 \mathrm{pp}$.

Modesto-Júnior, M. S.; Mascarenhas, R. E. B. 2001. Levantamento da infestação de Plantas Daninhas associadas a uma pastagem cultivada de baixa produtividade no Nordeste Paraense. Planta daninha, 19 (1): 11-21.

Murphy, S. D.; Clements, D. R.; Belaoussoff, S.; Kevan, P. G.; Swanton, C. J. 2006. Promotion of weed species diversity and re- 
duction of weed seedbanks with conservation tillage and crop rotation. Weed Science, 54: 69-77.

Paes, J. M. V.; Rezende, A. M. 2001. Manejo de plantas daninhas no sistema plantio direto na palha. Informe Agropecuário, 22: 37 42.

Pedrotti, D. E.; Guarim-Neto, G. 1998. Flora Ruderal da cidade de Cuiabá, Mato Grosso, Brasil. Acta Botanica Brasilica, 12 (2): 135-143.

Rodrigues, B. N.; Almeida, F. S. 2005. Guia de herbicidas. $5^{\mathrm{a}}$ ed. UFLA, Londrina, Brasil, 592pp.
Silva, A. A.; Silva, F. J. 2007. Tópicos em manejo de plantas daninhas. UFV, Viçosa, Brasil, 367pp.

Souza, V. C.; Lorenzi, H. 2005. Botânica sistemática: guia ilustrado para identificação das famílias de angiospermas da flora brasileira, baseado em APG II. Inst. Plantarum, Nova Odessa, Brasil, 640pp.

Souza, L. S.; Velini, E. D.; Maiomoni-Rodella, R. C. S. 2003. Efeito alelopático de plantas daninhas e concentrações de capim-braquiária (Brachiaria decumbens) no desenvolvimento inicial de eucalipto (Eucalyptus grandis). Planta Daninha, 21 (3): 343-354. 